\title{
Enquête
}

Archives de la revue Enquête

$2 \mid 1995$

Usages de la tradition

\section{Mémoire autobiographique et mémoire historique du passé éloigné}

Autobiographical memory and the historical memory of the more distant past

\section{Maurice Bloch}

Traducteur : Françoise Lacotte et Claude Lacotte

\section{OpenEdition \\ Journals}

\section{Édition électronique}

URL : http://journals.openedition.org/enquete/309

DOI : 10.4000/enquete.309

ISSN : 1953-809X

\section{Éditeur :}

Cercom, Éditions Parenthèses

Édition imprimée

Date de publication : 31 décembre 1995

Pagination : 59-76

\section{Référence électronique}

Maurice Bloch, « Mémoire autobiographique et mémoire historique du passé éloigné », Enquête [En ligne], 2 | 1995, mis en ligne le 10 juillet 2013, consulté le 10 décembre 2020. URL : http:// journals.openedition.org/enquete/309; DOI : https://doi.org/10.4000/enquete.309 


\title{
Mémoire autobiographique et mémoire historique du passé éloigné
}

\author{
Autobiographical memory and the historical memory of the more distant past
}

\section{Maurice Bloch}

Traduction : Françoise Lacotte et Claude Lacotte

1 La mémoire est à l'ordre du jour en anthropologie, en sociologie et même en histoire ${ }^{1}$. C'était jusqu'ici un domaine d'études réservé pour l'essentiel aux neurosciences et à la psychologie. Serait-ce à dire que s'amorce enfin entre ces disciplines une véritable collaboration intellectuelle, d'autant plus remarquable qu'elle porte sur une capacité humaine dont les manifestations relèvent effectivement d'approches caractérisant aussi bien les sciences expérimentales que les sciences humaines et sociales?

2 Cet optimisme doit vite être tempéré sitôt que l'on se penche sur ce que les spécialistes des unes et des autres écrivent à ce sujet. Tout se passe comme s'il n'y avait rien de commun entre eux, à l'exception de l'emploi d'un mot, dont la signification est difficilement spécifiable, et surtout comme s'ils avaient décidé de s'ignorer.

3 Les historiens, les anthropologues et les sociologues s'intéressent aux représentations du passé qui sont - ou plutôt passent pour être - transmises de génération en génération. Ils s'intéressent parfois aussi aux modalités de cette transmission: tradition orale, traces écrites, rites ou objets culturels. Les psychologues, à l'inverse, portent plus volontiers leur attention sur les mécanismes par lesquels s'effectuent, chez un individu, la mise en mémoire et la récupération de l'information, et non pas sur la nature du savoir qui a été emmagasiné, ni sur la façon dont il se transmet d'un individu à un autre. Et au cas où ce manque de corrélation n'apparaîtrait pas suffisamment, on observe que, comme le souligne U. Neisser dans un article important où il déplore le manque de contact entre ces disciplines $^{2}$, quatre-vingt-dix pour cent du travail des psychologues se rapportent à des souvenirs dont la durée n'excède pas une minute et qui, par conséquent, sont très 
éloignés des préoccupations des chercheurs en sciences sociales ou de toute personne intéressée par ce domaine.

En insistant sur ce point, Neisser souhaitait obtenir une coopération plus étroite entre les deux parties et on peut dire qu'il y est parvenu dans la mesure où, depuis quelque temps, l'intérêt des psychologues pour la mémoire à long terme " dans la réalité quotidienne ${ }^{3}$ " s'est considérablement développé et où il $\mathrm{y} \mathrm{a}$, de chaque côté, un réel désir de collaborer.

5 Toutefois il est rare que les bonnes intentions suffisent. Nous disposons maintenant d'ouvrages de psychologie, ceux de Neisser lui-même par exemple, et plus récemment ceux de Baddeley ${ }^{4}$ et Cohen - pour n'en citer que quelques-uns - qui comportent des références aux travaux de chercheurs en sciences sociales et d'historiens. De la même façon, nous trouvons les ouvrages de sociologues comme P. Connerton, d'anthropologues comme D. Holland et N. Quinn ou H. Whitehouse, et d'historiens comme M. Carruthers ${ }^{5}$, qui font référence à quelques-uns des travaux en psychologie concernant la mémoire à long terme. Mais, la plupart du temps, il semble qu'ils ne dépassent guère le stade de la simple mention et c'est comme s'ils avaient toutes les peines du monde à passer outre ces civilités préliminaires.

6 Nous tenterons dans cet article d'aller au-delà de ce premier stade. Dans une précédente étude $^{6}$, j'ai fait valoir que la prise de conscience du rôle de la mémoire dans la construction de la personne, de la part de psychologues tels que Nigro ou Neisser ${ }^{7}$, signifiait bien qu'ils devaient désormais tenir compte des travaux plus généraux des anthropologues sur la spécificité culturelle du concept d'individu en histoire. À présent, je voudrais démontrer, à l'inverse, que les anthropologues et les sociologues gagneraient à utiliser les travaux très techniques des psychologues s'ils souhaitent comprendre pleinement - or c'est, à l'évidence, un des objets essentiels de leurs recherches - la complexité que revêt la présence du passé conscient dans le présent. Je rejoins en cela un certain nombre d'anthropologues comme D. Sperber', qui ont, de la même façon, attiré l'attention de leurs collègues sur l'intérêt des travaux de certains psychologues, même si leurs points de vue et les miens sont différents sans être totalement incompatibles.

\section{Mémoire sémantique et mémoire autobiographique}

7 J'aimerais également défendre une idée qui, à première vue, pourrait sembler paradoxale: le souvenir qu'un sujet conserve de ce qu'il a vécu pendant sa vie - sa mémoire autobiographique - n'est pas d'une nature très différente de la connaissance qu'il a des événements historiques plus éloignés qu'il ne peut en aucun cas avoir vécus. J'utiliserai donc la distinction explicitement établie en psychologie par E. Tulving ${ }^{9}$ entre la mémoire autobiographique, ou épisodique, et la mémoire sémantique, même si cela doit me conduire par la suite à mettre l'accent sur leurs similitudes. La mémoire autobiographique se rapporte au souvenir des événements que le sujet a vécus - par exemple, quand il a appris à nager. La mémoire sémantique se rapporte aux faits que le sujet a appris par l'intermédiaire d'autres personnes, comme le nom de la capitale du New Hampshire. La mémoire sémantique comprend le souvenir de faits se rapportant au passé ; ainsi un sous-ensemble de la mémoire sémantique pourra s'appeler mémoire historico-sémantique, ou plus simplement mémoire historique.

8 Les psychologues, dans leurs travaux, traitent la mémoire autobiographique et la mémoire sémantique de façon très différente. Ils ont toujours montré un vif intérêt pour 
la nature des évocations liées à la mémoire autobiographique, et pour la relation qu'elle entretient avec la perception du moi. En particulier, ils ont souligné l'opposition entre le fait de "se souvenir » et celui d'« évoquer ». On évoque le passé soit en présence d'autres personnes - présence réelle ou imaginée -, soit au cours de monologues silencieux ou oralisés. Il n'en reste pas moins que le souvenir de ce qu'on a vécu est beaucoup plus riche que ce qu'on en évoque, beaucoup plus, en vérité, que ce dont on a conscience, comme le démontre l'épisode de la madeleine chez Proust, ou plus communément, comme en témoigne la capacité que nous avons de nous apercevoir très vite que nous avons déjà lu un livre, dès les premières pages, malgré l'impression que nous avions, quelques instants auparavant, de n'en avoir gardé aucun souvenir. Certains psychologues soutiennent même que rien n'est complètement oublié qui, dans certaines circonstances, ne puisse être évoqué; et de nombreuses études expérimentales ont démontré comment des souvenirs dont les sujets ignoraient l'existence pouvaient être ramenés à la surface si l'on provoquait certains états affectifs. Il s'ensuit que la mémoire autobiographique est à la fois très présente et très difficile à isoler.

Pour ce qui est des ouvrages des psychologues sur la mémoire sémantique - à l'exception de quelques-uns dont il sera question plus loin -, ils se sont rarement intéressés à la nature de son contenu et à sa relation avec le concept de personne ou d'identité. Ils se sont presque exclusivement interrogés sur les mécanismes de la réminiscence et de l'oubli.

10 Il est intéressant de constater que cette opposition rejoint celle qui est établie traditionnellement par les historiens entre mémoire et histoire. Quand les historiens parlent de mémoire, ils se réfèrent à ce que des sujets se rappellent de ce qui leur est arrivé au cours de leur vie ; et comme ils s'intéressent à ce que ces souvenirs peuvent leur apprendre sur le passé, ils ont une conscience aiguë du caractère labile et multiforme de ce phénomène - caractère qu'ils peuvent considérer comme un inconvénient ${ }^{10}$ ou une source de richesses ${ }^{11}$. Quand ils se penchent sur un passé éloigné, ils perdent tout intérêt pour les problèmes psychologiques de la connaissance et ne retiennent que le degré d'efficacité et de précision qu'apportent des modalités de transmission telles que la tradition orale ou les documents.

11 Je m'intéresserai, pour ma part, à l'histoire en tant que fait social dans la vie des individus; et tout comme j'entends montrer que, dans cette perspective, la différence entre mémoire autobiographique et mémoire historico-sémantique est bien moindre que ce qu'on pourrait imaginer, de la même façon je chercherai à savoir si cette différence entre histoire et mémoire est tout aussi nette qu'on a pu le faire croire.

12 En adoptant une telle position, je me rapproche et m'écarte à la fois des travaux admirables et éclectiques de M. Halbwachs ${ }^{12}$ sur la mémoire. Halbwachs voulait surtout, lui aussi, essayer de rendre le côté subtil, irréductible et multiforme qui caractérise d'une manière à la fois explicite et implicite - la présence du passé au sein du présent ; et il y parvient superbement. Il souhaitait également insister, à juste titre, sur le caractère social de la mémoire - puisque l'évocation implique une communication avec les autres -, et aussi sur le fait que, dans la mesure où le souvenir individuel est sans cesse soumis à des transformations et à des reformulations au cours de ce processus de communication, il perd par là même son caractère isolé, indépendant et individuel. C'est ainsi que, pour Halbwachs, et comme nous le montrerons nous-même plus loin, des souvenirs se référant à des périodes bien antérieures à la vie du sujet peuvent partager beaucoup de caractéristiques de la mémoire autobiographique. 

la distinction entre "évoquer " et "se rappeler ", Halbwachs va jusqu'à nier toute fonction épistémologique dans les mécanismes de fonctionnement du cerveau humain. Une telle position l'amène à soutenir des affirmations manifestement erronées : ainsi, selon lui, des souvenirs qui ne sont pas partagés sont vite oubliés. Il n'y a donc, à ses yeux, aucune différence entre la mémoire autobiographique et la mémoire collective, puisque la mémoire autobiographique est aussi le produit d'un contact social. Cet amalgame des deux types de mémoire le conduit également à opposer, de façon radicale, la mémoire, qu'il pose comme subjective même si elle est collectivement subjective, à l'histoire, qui serait, elle, objective et apparemment indépendante du contexte social dans lequel elle est produite ou consommée. Plus grave encore, il élude le problème de la pensée du sujet en tant que facteur actif dans la transmission du passé, ce qui le conduit à traiter le processus et la nature même de la mémoire comme un phénomène indéterminé et flou, chose étrange pour une étude qui était censée porter sur ce thème.

Dans ce qui suit, je voudrais montrer, à la lumière d'une étude de cas spécifique, qu'il est possible de retenir et de poursuivre les analyses particulièrement judicieuses faites par Halbwachs, mais je voudrais aussi montrer qu'en faisant abstraction de l'antipathie - empruntée à Durkheim - qu'il éprouve pour la psychologie, nous pouvons saisir beaucoup plus clairement qu'il ne le fait, les processus sociaux qu'il entendait expliquer.

\section{Souvenirs du soulèvement de 1947 à Madagascar}

15 Afin de démontrer l'intérêt de faire appel aux observations tirées de la psychologie cognitive, pour les mettre au service des phénomènes relevant par excellence du champ des sciences sociales, je voudrais revenir sur l'exemple de Madagascar, qui m'a déjà servi lors d'articles précédents, mais pour en tirer, cette fois, des conclusions sensiblement différentes.

Il s'agit d'un village de cultivateurs sur brûlis dans la forêt de l'est de Madagascar, que j'observe depuis maintenant presque vingt-cinq ans. En 1947, le village fut complètement brûlé par les troupes françaises, à titre de représailles contre les habitants dont on présumait qu'ils avaient soutenu le soulèvement dirigé contre l'autorité coloniale qui avait eu lieu cette année-là. Une période de plus de deux ans succéda à cet acte de violence, au cours de laquelle les villageois se dissimulèrent dans une forêt, dans des conditions de vie extrêmement difficiles, de crainte de se faire capturer par les forces françaises.

Lors d'un premier article ${ }^{13}$, j'ai examiné ce qu'on pourrait nommer le récit officiel de cette période de traumatisme, tout particulièrement l'épisode du retour au village des habitants apeurés. Il s'agissait là d'un récit classique qui mêlait, sans solution de continuité, des thèmes symboliques traditionnels et des concepts catholiques, pour aboutir à ce qu'on pourrait appeler une version « canonique ». Ce furent trois anciens du village qui, après une longue délibération, prirent la décision de m’informer de ce récit ; ils savaient que je souhaitais obtenir des informations sur les événements qui s'étaient produits, mais ils s'étaient montrés jusque-là peu disposés à en parler à un Européen. En fait, les anciens racontaient souvent cette histoire à leurs compagnons, au cours des veillées par exemple, et c'est là que, depuis, je l'ai entendue raconter presque mot pour mot. 
C'est un retour au village qui donna lieu au deuxième article ${ }^{14}$, alors que le premier, déjà terminé, était en cours de publication. M'étant trouvé bloqué, pendant plusieurs heures, dans une case en brousse avec un des survivants du soulèvement - précisément celui qui, à l'origine, m'avait fait part de la version officielle-, j'ai découvert une version totalement différente des événements, même si elle ne contredisait pas la version canonique. Ce nouveau récit reposait bien plus sur des faits et n'avait pas le caractère mythologique intemporel du premier. Il était largement inspiré par le fait que, de la hutte, il était possible d'apercevoir une vallée où des événements marquants s'étaient déroulés au temps du soulèvement. Le sujet semblait en mesure de se représenter ce qui s'était passé durant ces moments terribles, alors même qu'il observait le paysage qu'il avait devant les yeux.

Au plan théorique, j'ai tiré de cet incident les conclusions suivantes : nous ne devons, en aucun cas, confondre un récit d'un événement avec le souvenir qu'en ont gardé les participants. Un récit se fonde sur le souvenir; néanmoins les sujets peuvent très bien solliciter leurs images mentales pour élaborer d'autres récits lorsqu'ils sont stimulés par un contexte social ou, dans le cas précédent, un contexte visuel. Ce qui constitue le souvenir ne se résume pas à un récit en particulier, ni même à la somme de tous les récits; il est conservé sous une forme non verbalisée, et son contenu reste incertain, même pour les principaux intéressés. Ainsi on aurait dit que cet homme qui évoquait le détail des événements dans la case découvrait, au fil du récit, de plus en plus de détails.

Dans un certain sens, l'expérience ne démontrait que trop bien combien l'évocation et le souvenir sont deux choses tout à fait distinctes. La portée anthropologique d'une telle observation est considérable, puisque la présence du passé au sein du présent est bien plus complexe, bien moins explicite, mais peut-être bien plus puissante que l'existence de récits explicites ne pourrait nous le faire croire. Ceci suggère que des sujets sont susceptibles de n'avoir pas conscience de ce dont ils se souviennent, comme dans le cas précédent et, par conséquent, de ne pas avoir conscience de l'importance de ce que représente pour eux ce souvenir, comme voudrait nous le faire croire la psychanalyse. Néanmoins, un très grand nombre de travaux nettement moins contestables en psychologie cognitive, étayés par d'importantes études expérimentales, ont montré la capacité d'un sujet à évoquer des souvenirs d'une diversité incroyablement riche. Les chercheurs en sciences sociales seront tout particulièrement intéressés de savoir qu'on peut retrouver certains souvenirs qui paraissaient complètement perdus, lorsque le sujet concerné se remet dans la situation émotionnelle dans laquelle il était au moment où les faits ont eu lieu ${ }^{15}$. Ceci signifie que le passé se présente comme une réserve de matériaux toujours adaptables selon les situations ou les dispositions d'esprit qui sont souvent le résultat de contextes sociaux organisés ${ }^{16}$.

\section{De la mémoire autobiographique à la mémoire historique}

21 Tout en accordant une certaine importance aux considérations d'ordre psychologique pour ce qui concerne la mémoire autobiographique, on peut toutefois considérer que ce type d'informations n'a qu'une portée limitée. Après tout, la distinction entre " évoquer " et « se souvenir », sur laquelle elles reposent, ne paraît s'appliquer qu'au souvenir de faits qu'on a vécus, puisque, dans le cas de l'évocation d'un passé plus lointain, les 
informateurs ne s'appuient que sur des récits qu'ils tiennent d'autres individus, et auxquels il manque le caractère multiple du souvenir des faits auxquels on a été mêlé. En d'autres termes, lorsqu'on a affaire à ce type de souvenirs historiques, il ne s'agit que de la mémoire sémantique; et les seules considérations d'ordre psychologique qui soient pertinentes sont celles qui concernent la fiabilité - ou la non-fiabilité - des différents procédés mnémoniques utilisés pour retenir des récits déjà construits que les informateurs tiennent d'autres individus.

Une telle conclusion, malgré son apparent bon sens, est, selon moi, erronée ; et pour le démontrer, je présenterai, tout d'abord, ce qui est arrivé lors d'une autre visite à ce même village et qui avait fait l'objet des deux autres articles.

En septembre 1995, j'ai été conduit par un groupe de villageois à l'endroit même où s'étaient cachés les habitants en 1947. J'étais accompagné de la personne qui avait été mon principal informateur pour ce qui est des souvenirs exposés dans mes articles précédents, ainsi que d'environ dix membres de sa famille, dont plusieurs de ses petitsenfants et même un de ses arrière-petits-enfants. Le fait de nous retrouver à cet endroit a offert l'occasion de revenir longuement sur les histoires qu'on m'avait déjà racontées et d'ajouter des éléments suscités en partie par la topographie, en partie par les traces que nous avions retrouvées. Bien évidemment, c'était cette personne qui constituait ma principale source d'informations, m'expliquant en détail à quoi ressemblaient leurs maisons, comment ils avaient réussi à préparer leur nourriture, où ils cachaient leurs maigres provisions, comment ils surveillaient l'armée sans être vus... Cependant, le plus étonnant, peut-être, était que tous les membres de la famille, y compris les enfants, me montraient ce qu'ils avaient fait à cette époque, où ils s'étaient réfugiés, la manière dont ils avaient vécu les événements, alors qu'à l'évidence ils n'étaient pas nés en 1947. Le fait de se trouver dans ce lieu créait donc bien une atmosphère qui offrait l'occasion d'une conversation générale et d'une répétition de choses connues, à laquelle tous semblaient prendre part, non seulement, semble-t-il, pour nous informer, mais pour raconter et exprimer en détail ce que chacun d'eux savait.

À vrai dire, il n'y avait rien là de bien mystérieux. Ce qui s'était passé, c'est que tous les membres de la famille avaient entendu dire beaucoup de choses à propos de ces événements et s'étaient probablement rendus de nombreuses fois sur le site; par conséquent, ils savaient à quoi s'en tenir. En parlant de cette période, tous, les jeunes comme les vieux, utilisaient le pronom «nous ». L'imprécision qu'autorise cette forme est significative. Cela pouvait signifier qu'ils avaient été présents en personne, ou simplement que "notre famille» avait été là. Je pense que cette rhétorique de l'énonciation sous-entendait, en fait, qu'il n'était pas nécessaire, à leurs yeux, de faire un choix, encore que, selon toute vraisemblance, si je leur avais posé la question, ils auraient reconnu qu'ils n'étaient pas présents physiquement à l'époque. En réalité, mes informateurs utilisaient non pas le «nous » qui, en langue malgache, exclut la personne à qui l'on s'adresse, mais un «nous» qui inclut le destinataire du discours, moi en l'occurrence. Ce faisant, ils ne supposaient pas que j'avais été présent, pas plus que les enfants, mais ils marquaient par l'usage de ce pronom que je faisais partie de la famille, ce que font souvent les Malgaches, en partie parce que le «nous » exclusif est souvent perçu comme une forme inconvenante, mais aussi pour marquer la continuité qui existe entre des gens que rapproche un lien moral. Ils auraient facilement perçu le sens de la phrase de John Donne « Ne demande pas pour qui sonne le glas ${ }^{17} »$. 
S'il est vrai qu'il n'y a rien là qui doive surprendre, ceci n'en donne que plus de poids au phénomène, en montrant bien que de telles situations sont monnaie courante.

On voit là, entre autres choses, le rôle joué par les objets matériels dans ce mode de transmission. La topographie, lorsqu'elle est, comme dans le cas qui nous occupe, lourde d'histoire, est une composante particulièrement signifiante dans la mesure où elle permet au sujet de se réapproprier plus facilement l'événement comme s'il en avait vraiment été le témoin. Le rôle des paysages chargés d'histoire est quelque chose que les historiens, les sociologues et les anthropologues ont souvent discuté18. Schmitt a aussi montré, en étudiant une tradition locale se rapportant à un bois sacré, comment le lien entre les lieux et le récit produit une transmission à long terme qui conserve une surprenante exactitude ${ }^{19}$. Notre compréhension du pouvoir qu'ont les objets et les lieux de générer des images a été mise en lumière par les psychologues qui s'intéressent aux modes de fonctionnement de la mémoire ${ }^{20}$ et leurs travaux montrent que ce n'est pas le fruit du hasard si la mémoire sociale à long terme, qui produit des images, s'appuie autant sur des visites à des sites sanctifiés par le souvenir d'événements qui sont censés s'y être déroulés.

Cependant ce que m'apportait surtout le fait que tous, jeunes et vieux, se rappelaient à quoi ressemblait l'endroit où ils s'étaient cachés, c'était la preuve éclatante qu'un passé que vous n'avez pas personnellement vécu, soit parce qu'il remonte à une date antérieure à votre naissance, soit parce que vous n'avez pas assisté à l'événement, conserve en vous une présence qui va au-delà du simple souvenir des récits qu'on a pu vous en faire. Les enfants qui me faisaient visiter le site du village, et moi aussi à dire vrai, imaginions le passé, mais ce passé était lié à une expérience affective et empirique immédiate qui ressemblait à celle que nous aurions éprouvée si nous avions vécu l'événement. Ce qui se fixait dans notre esprit, c'était bien un modèle mental, fait à la fois d'images et d'émotions et qui était, par conséquent, susceptible d'être sollicité de la même manière que les souvenirs autobiographiques.

Ceci ne veut pas dire que l'information conservée par les enfants était de même nature que celle des gens qui avaient vécu le traumatisme des événements de 1947. Cette hypothèse serait hautement improbable ; mais on peut dire aussi que cette information n'était pas radicalement différente, puisque toute version par trop fantaisiste de ces événements pouvait être soumise au contrôle né d'une continuité de contacts entre les générations, comme l'a bien montré Halbwachs. Surtout, cela constituait pour eux une expérience " vécue ». Leur souvenir de cette période où les villageois avaient vécu cachés, n'était donc pas d'une nature fondamentalement différente de celui qu'en conservaient ceux qui l'avaient vécue.

On ne prend pleinement conscience de ce que signifie cette observation que si l'on considère ses implications pour la transmission à long terme du passé. Si l'on pense aux enfants qui m'accompagnaient sur le site, on voit bien qu'ils pourront transmettre le souvenir du soulèvement d'une manière assez semblable à celle par laquelle ce souvenir leur a été transmis, c'est-à-dire non pas en récitant une fois de plus ce qu'on leur avait raconté, mais en réimaginant des événements qui, à la fois, faisaient et ne faisaient pas partie de leur expérience, et en construisant des récits fondés sur des informations accumulées dans leur mémoire, accumulées, nous commençons à le comprendre, comme peuvent l'être dans notre cerveau les traces du passé ${ }^{11}$. De telles évocations se produiront de manières diverses et dans des lieux variés, mais il est très probable que ce sera le plus souvent sur le site même où nous étions rassemblés en présence d'autres, qui seront alors 
en mesure de perpétuer le processus de transmission d'une expérience appartenant à un passé lointain et pourtant quasi autobiographique.

\section{La tradition orale comme mémoire autobiographique} dans un lieu particulier ne se limite pas à ce mode de transmission; il se produit également lorsqu'il n'existe comme support pour le souvenir évoqué que la tradition orale et la tradition écrite. avec la mémoire autobiographique dont nous avons identifié l'existence dans le cas de la transmission des événements de 1947, ne peut exister, et que dans de tels cas, la seule question pertinente est de savoir jusqu'à quel point les sujets se souviennent des histoires qui leur ont été racontées et comment les conteurs traditionnels parviennent à accomplir ces prouesses de mémoire que l'on a souvent constatées ${ }^{22}$. De la même manière, la plupart des études portant sur la mémoire sémantique qui s'appuient sur les travaux des psychologues, même si elles reposent sur des fondements méthodologiques très différents, s'intéressent elles aussi principalement à l'exactitude de la mémorisation des faits ou à son inexactitude. C'est ainsi que D. Rubin, dans son étude sur la psychologie cognitive de la transmission orale - sans doute l'une des études les plus détaillées sur la question - pose encore une fois les questions classiques portant sur l'évocation de l'information sémantique. Bien qu'il étudie longuement le sens que revêt l'évocation des images visuelles dans le processus de mémorisation des récits, il n'y voit qu'un support à l'interprétation du texte et ne se demande pas dans quelle mesure la présence de ces images visuelles agit comme une alternative à la mémorisation des textes, ni quels sont les liens qu'elle entretient avec la mémoire autobiographique, ni enfin en quoi elle affecte les textes eux-mêmes ${ }^{23}$.

Il existe quelques exceptions à ce manque d'intérêt pour le contenu de la mémoire sémantique. Ainsi D. Sperber avance que la nature des récits dont on se souvient est déterminée, en partie, par des prédispositions innées de l'esprit humain qui agit ainsi, avec le temps, comme une sorte de filtre qui sélectionne les histoires intéressantes ; c'est ainsi qu'il justifie la présence de certains contenus, de préférence à d'autres. Il faut dire qu'on est très loin d'avoir démontré une théorie aussi audacieuse.

Il existe une position presque inverse, bien que compatible, qui remonte au psychologue de Cambridge Frederick Bartlett, qui suggère dans son livre Remembering ${ }^{24}$ que le mécanisme de rétention des récits ressemble bien plus à celui des épisodes autobiographiques qu'on ne pourrait le penser à première vue. Bartlett s'intéressait à la mémorisation des récits et, par conséquent, à la manière dont étaient conservées en mémoire de telles connaissances. Il a utilisé des observations anthropologiques pour montrer comment la mémorisation d'un mythe amérindien par de jeunes étudiants de Cambridge ne pouvait s'accomplir facilement que lorsqu'ils l'avaient transformé de telle manière qu'il s'accordait avec leur culture, ou pour reprendre le terme introduit par Bartlett, un "schéma " de leur culture. Ce qu'il y a ici de particulièrement important, c'est que, d'après les conclusions de Bartlett, ce n'est pas le récit lui-même qui est mis en mémoire, mais une représentation non verbalisée, inexplicite, mais dont on se souvient aisément, des événements rapportés par le récit. 

existe une théorie plus élaborée concernant des observations de ce type : celle qui repose sur la notion de modèles mentaux, développée par Johnson-Laird, et qu'on peut considérer comme une extension de la théorie des schémas de Bartlett. L'auteur considère, et démontre en s'appuyant sur de nombreuses observations expérimentales, que, dans des conditions normales, se souvenir d'une histoire, c'est construire un modèle mental cohérent qui vous permet de vous rappeler ce qui s'est passé comme si ces événements se déroulaient devant vos yeux ; c'est cet événement imaginé et non le texte qui reste dans la mémoire. De plus, cet « effort pour produire du sens ", pour reprendre la terminologie de Bartlett, signifie que les éléments qui ne sont pas contenus dans le texte sont inférés pour rendre la compréhension possible, idée que reprennent à leur compte avec force Sperber et Wilson dans leur ouvrage sur la pertinence et son importance pour la compréhension du langage et qui est encore affinée dans la critique que fait Sperber de Johnson-Laird, dans laquelle il montre les insuffisances des critères régissant l'apparition de telles inférences ${ }^{26}$.

Ce point est, me semble-t-il, très important pour notre compréhension de la nature des récits historiques qui sont, d'une manière profonde, constitutifs de l'identité et de la vie morale des individus, comme c'est le cas de ceux qui font l'objet de notre discussion. On peut penser qu'à cause de leur valeur profonde, ces récits entendus dans l'esprit de ceux qui les retiennent, font l'objet de très importants étoffements par inférence, à tel point que leur contenu empirique d'un récit, inévitablement ténu, est remplacé par une 
expérience qui est pratiquement de même nature que s'il s'agissait d'une expérience vécue. Il devient alors possible pour les sujets d'« évoquer » des aspects du passé qu'ils ont appris dans un récit qui ne contenait aucune information de ce type, mais qu'ils ont inférés pour lui donner du sens. Cela veut dire qu'en dépit de la pauvreté de l'input d'origine, ils peuvent, quand ils veulent se rappeler, scruter une mémoire vive et presque illimitée des événements contenus dans le récit, exactement comme pourrait le faire quelqu'un qui évoque des souvenirs autobiographiques. C'est ainsi que pour le récit d'événements de grande importance pour les sujets concernés, la différence de nature, sinon de contenu, entre la mémoire historique et la mémoire autobiographique disparaît complètement.

\section{La mémoire autobiographique comme mémoire historique}

Si l'on peut dire que le souvenir né des récits peut prendre la forme de souvenirs autobiographiques, il existe aussi des éléments qui prouvent que l'évocation de souvenirs autobiographiques liés à des expériences remontant à un passé lointain peuvent ressembler à l'évocation d'événements qui sont arrivés à d'autres et dont on a seulement entendu parler.

Les travaux portant sur la mémoire autobiographique suggèrent que lorsque les sujets évoquent des événements dont ils ont été eux-mêmes témoins ou auxquels ils ont participé, ils se souviennent de ces événements comme s'ils avaient été extérieurs à euxmêmes. Ainsi, Nigro et Neisser ont établi que les sujets ressentaient leurs souvenirs d'événements lointains comme des expériences extérieures, comme s'ils n'avaient pas véritablement été impliqués dans ce qui s'était passé mais n'en avaient été que de simples spectateurs $^{27}$. Une telle impression signifie que certains souvenirs autobiographiques ressemblent plutôt à des souvenirs historiques d'événements qui auraient été rapportés au sujet par une tierce personne.

Un autre aspect des souvenirs autobiographiques est que, au moment de leur évocation, il est possible, et même probable, que celui qui parle ne se souvienne pas de l'événement lui-même, mais du dernier récit qu'il en a fait. Ainsi, dans le cas de l'histoire que j'ai analysée dans le premier des trois articles, il est hautement probable que les anciens qui me l'ont racontée l'avaient racontée à plusieurs reprises; ils ne faisaient donc qu'en partie revivre le passé ; ils se rappelaient surtout des circonstances semblables où ils avaient déjà raconté leurs histoires. Dans un cas semblable, il n'y a pratiquement aucune différence entre l'histoire orale au sens où on l'entend communément et l'évocation d'un tel «souvenir autobiographique». Dans le cas du récit de 1947 étudié dans le second article, cette hypothèse est improbable, car je crois que l'homme qui me l'a raconté ne l'avait jamais fait auparavant et je pouvais presque voir « surgir le passé » devant ses yeux à mesure qu'il parlait. Ici, la différence avec la mémoire sémantique apparaissait clairement: les aspects expérientiels, empiriques et affectifs pouvaient être retrouvés d'une manière presque intarissable. Dans la majorité des cas nous avons affaire à quelque chose d'intermédiaire, mais c'est précisément ce que je veux démontrer: lorsqu'on commence à étudier la mémoire dans le monde réel, on s'aperçoit que la mémoire autobiographique et la mémoire historique se rejoignent. 


\section{L'écrit et la mémoire}

41 Lorsqu'intervient l'écrit, on pourrait penser que la séparation entre un récit du passé et une expérience individuelle est d'ordre absolu puisque le mécanisme de conservation n'a plus rien à voir avec une forme quelconque de la mémoire humaine. Lorsque le passé est conservé sous forme explicite dans des documents, des livres et autres artefacts, tout élément se rapportant à l'imagination et à l'organisation non linguistique de la connaissance est exclu. Et pourtant, même sur ce point, les choses ne sont pas aussi tranchées. Pour qu'un écrit acquière un statut historique, il a besoin d'être lu et, la plupart du temps, d'être l'objet d'une réécriture. Il n'y a aucune raison de penser que le processus qui consiste à conserver en mémoire un récit dont on a pris connaissance par l'écrit est tant soi peu différent de ce qu'il est pour un autre dont on a pris connaissance en l'écoutant. Les mêmes processus psychologiques entrent donc en ligne de compte.

On peut remarquer que l'étude des fans de Sherlock Holmes que j'ai évoquée plus haut concernait en fait un texte écrit qui est conservé dans la mémoire du lecteur selon des modes qui impliquent une représentation imaginaire des événements peut-être comparable, dans une certaine mesure, à ce qu'elle aurait été s'ils avaient été présents. Le fait que ces fans avaient eu connaissance des histoires par voie écrite et non par un récit n'est pas pertinent, ce qui ne signifie pas, naturellement, que ceci ait une incidence sur la stabilité à long terme de leur contenu. Il est donc tout à fait possible qu'une histoire que l'on a apprise par l'intermédiaire de l'écrit puisse créer des modèles mentaux dans lesquels le phénomène d'inférence joue un rôle nécessaire dans la compréhension et dans la conservation du souvenir. La preuve qu'il peut en effet en être ainsi nous est fournie par le groupe le plus orthodoxe qui soit, ceux qui, normalement, considèrent qu'il leur appartient de créer des récits qui s'appuient uniquement sur des sources documentaires, presque exclusivement écrites : je veux parler des historiens. C'est ainsi que G. Duby, le spécialiste du Moyen-Âge, écrit à propos des soldats qui ont combattu à la bataille de Bouvines au XIII ${ }^{\mathrm{e}}$ siècle : «Je les observais comme Margaret Mead avait observé les Manus. Aussi désarmé qu'elle, mais pas plus ${ }^{28}$.»

43 Ce qui semble donc se passer, dans le cas de la tradition historique écrite lorsque les gens la considèrent comme suffisamment importante pour en conserver trace dans leur mémoire, c'est que nous avons affaire à un phénomène de flux continu dans lequel les modèles mentaux du passé, peut-être construits en partie à partir de documents, mais ayant la richesse d'évocations quasi autobiographiques, deviennent source d'autres récits écrits qui se distinguent par leur caractère explicite mais aussi par leur faible pouvoir d'évocation; ces derniers prendront place à leur tour dans la construction de modèles mentaux que viendront compléter, pour une large part, les inférences construites par des individus. Il paraît donc établi que pour tout un chacun, et non pas seulement pour les historiens, les épisodes du passé sont conservés dans le souvenir selon des modes qui entretiennent une ressemblance frappante avec ceux qui concernent les événements que l'on a soi-même vécus.

Le passage du passé au présent doit inclure une compréhension de la manière dont les individus sont affectés par leur propre mémoire épisodique. Les recherches en sciences cognitives ne peuvent donc être écartées par les chercheurs en sciences sociales. Le souvenir du passé ne se limite pas à une simple «représentation collective ». Ceux qui voudraient nous faire penser les choses de cette façon sont amenés inexorablement à 
adopter la position caractéristique des anthropologues qui accentue à juste titre l'évocation publique ${ }^{29}$ mais oublie que ce qui n'est pas exprimé n'est pas nécessairement oublié et possède de ce fait une signification sociale puisqu'il s'agit d'une ressource mise en réserve pour de futures représentations sociales. Ceci étant dit, nous ne devons pas pour autant négliger l'argument d'Halbwachs: le souvenir est aussi un phénomène social ; c'est ainsi qu'il est transmis. Nous avons vu cela dans l'épisode du retour des villageois de Madagascar à l'endroit où ils s'étaient cachés. Toutefois, n'en déplaise à Halbwachs, ce que nous avons vu également, c'est qu'une telle transmission requiert pour être comprise de se référer à des processus psychologiques individuels, ceux-là mêmes qu'il cherchait à exclure.

\section{NOTES}

1. Je tiens à remercier G. Lenclud de l'aide qu'il m'a apportée lors de la rédaction de cet article.

2. U. Neisser, "Memory: What are the Important Questions?", in M. M. Gruneberg et al., Practical Aspects of Memory, Londres, Académie Press, 1978.

3. G. Cohen, Memory in the Real World, Hove, Lawrence Erlbaum Associates, 1990.

4. A. Baddeley, Human Memory: Theory and Practice, Hove, Lawrence Erlbaum Associates, 1990.

5. P. Connerton, How Societies Remember, Cambridge, Cambridge University Press, 1989; D. Holland, N. Quinn, Cultural Models in Language and Thought, Cambridge, Cambridge University Press, 1987 ; H. Whitehouse, « Memorable Religions : Transmissions, Codifications and Change in Divergent Melanesian Contextes », Man, 27, 1992 ; M. Carruthers, The Book of Memory: A Study of Memory in Medievial Culture, Cambridge, Cambridge University Press, 1990.

6. M. Bloch, «Internal and External Memory : Different Ways of Being in History ", in Suomen Antropologi (Helsinki), 1992.

7. G. Nigro \& U. Neisser, « Points of View in Personal Memory », Cognitive Psychology, 15, 1983.

8. D. Sperber, «Anthropology and Psychology : Towards an Epidemiology of Representations ", Man, 20, 1985.

9. E. Tulving, "Episodic and Semantic Memory», in E. Tulving, \& W. Donaldson, eds, The Organisation of Memory, New York, Academic Press, 1972.

10. S. Courtois, "Archives du communisme : mort d'une mémoire, naissance d'une histoire », Le Débat, 77, 1993.

11. T. Todorov, «La mémoire devant l'histoire », Terrain, 25, 1995.

12. M. Halbwachs, Les cadres sociaux de la mémoire, Paris, Presses universitaires de France, 1925, et La Mémoire collective, Paris, Presses universitaires de France, 1950.

13. M. Bloch, «The Resurection of the House ", in J. Carsten \& S. Hugh-Jones, eds, About the House, Cambridge, Cambridge University Press, 1995 (l'article a été écrit en 1989).

14. M. Bloch, «Time Narratives and the Multiplicity of Representations of the Past », Bulletin of the Institute of Ethnology (Taïwan), 75, 1993.

15. A. Baddeley, op. cit., chap. $12,15$.

16. S. G. Gillingham \& G. H. Bower, « Cognitive Consequences of Emotional Arousal », in C. Izard et al., eds, Emotions, Cognition and Behaviour, Cambridge, Cambridge University Press, 1984.

17. Poème de John Donne (1573-1631) rendu célèbre par le roman de Hemingway, Pour qui sonne le glas. Le poème dit, en substance : aucun être humain n'est une île isolée; chacun fait partie de 
l'unique continent des hommes ; «La mort de tout homme me diminue personnellement parce que je fais partie du genre humain. N'envoie donc pas demander pour qui sonne le glas. C'est pour toi qu'il sonne. » [N.d.T.]

18. Cf. D. Lowenthal, The Past is a Foreign Country, Cambridge, Cambridge University Press, 1985 ; P. Commerton, op. cit.; P. Nora, ed., Les lieux de mémoire, Paris, Gallimard, 1984-1992, 7 vol.; M. Bloch, « People into Places : Zafimaniry Concepts of Clarity », in E. Hirsch \& M. O'Hanlon, The Anthropology of Landscape, Oxford, Oxford University Press, 1995.

19. J.-C.Schmitt, Le Saint lévrier: Guinefort, guérisseur d'enfants depuis le XIII siècle, Paris, Flammarion, 1979.

20. A. Baddeley, op. cit., chap. 5.

21. P. N. Johnson-Laird, Mental Models, Cambridge, Cambridge University Press, 1983, chap. 15.

22. M. Parry \& A. B. Lord, eds, Serbocroatian Heroic Songs, Cambridge, Harvard University Press, 1954, vol. 1.

23. D. Rubin, Memory in Oral Traditions, Oxford, Oxford University Press, 1995.

24. F. Bartlett, Remembering, Cambridge, Cambridge University Press, 1932.

25. U. Neisser \& J. Hupsey, « A Sherlockian Experiment », Cognition, 3, 1974.

26. D. Sperber \& D. Wilson, La pertinence. Communication et cognition, Paris, Éd. de Minuit, 1989 (éd. originale : Oxford, Blackwell, 1986); D. Sperber et al., « Relevance Theory Explains the Selection Task », Cognition, 1996.

27. G. Nigro \& U. Neisser, op. cit.

28. G. Duby, L'histoire continue, Paris, Odile Jacob, 1991, p. 156.

29. L. Bohannan, «A Genealogical Charter», Africa (Edimbourg), 22, 1952; M. Kilani, La Construction de la Mémoire, Genève, Labor \& Fides, 1992.

\section{RÉSUMÉS}

La psychologie et les sciences sociales traitent la mémoire de façon très contrastée : cet essai compare leurs approches et montre en particulier que les caractéristiques de la mémoire autobiographique (ou épisodique) - c'est-à-dire son caractère non explicite, le fait que le souvenir puisse être évoqué de façon toujours nouvelle, la charge émotionnelle qu'il contient et la multiplicité d'aspects qu'il revêt - peuvent aussi s'appliquer à la mémoire d'un passé plus lointain dont les individus n'ont pas fait l'expérience directe. Ceci tient au fait que les évocations associées à des lieux ou à des objets transforment les récits qu'on a entendus d'autres personnes en expériences personnelles: les récits sont nourris par ceux qui les entendent à partir de processus inférentiels, en sorte que des événements dont on a seulement entendu parler peuvent faire l'objet de représentations aussi vives que le souvenir d'expériences personnelles. Le point de vue présenté dans cet article est illustré par un exemple tiré d'une recherche ethnologique menée à Madagascar.

The article concerns the contrasting approaches to memory found in psychology and the social sciences. In particular it shows that the characteristics of autobiographical or episodic memory, its lack of explicitness, the fact that the remembered can be recalled in ever new ways, its emotional charge and its multiplicity of aspects can also apply to memories of a more distant past which individuals have not experienced directly. This is because evocations linked to places or objects transform narratives heard from others into personal experiences and because 
narratives are always fleshed out by those who hear them through various inferential processes, so that past events about which one has only heard about may gain the same vividness as memories of personal experiences. The argument is illustrated by a case from Madagascar. 